\title{
Bilateral Calcified Chronic Subdural Hematoma in a Patient with Ventriculoperitoneal Shunt: A Case Report
}

\author{
Yo Seob Shin, Young Woo Lee, Dong Gyu Shin, Woo Ram Shin \\ Department of Neurosurgery, Presbyterian Medical Center, Jeonju, Republic of Korea
}

Corresponding author: Young-Woo Lee Department of Neurosurgery, Presbyterian Medical Center, 365, Seowon-ro, Wansan-gu, Jeonju 54987, Republic of Korea Tel: $+82-63-230-1420$

Fax: $+82-63-230-1429$

E-mail: yyong0411@naver.com

Received: August 15, 2019

Revised: September 10, 2019

Accepted: September 15, 2019
Chronic subdural hematoma (CSDH) is a common condition. However, it is rarely calcified. Among various causes, hydrocephalus and ventriculoperitoneal shunts in CSDH are uncommon causes of calcification. A 38-year-old woman presented with visual disturbance. Computed tomography and magnetic resonance imaging of the brain demonstrated compressed optic chiasm by planum sphenoidale meningioma. And massive bilateral calcified CSDH with cerebral convexity. Surgery was performed to remove the tumor and ipsilateral calcification. The symptoms improved following discharge after surgery. As this is a rarely performed surgery, caution is advised to prevent damaging the dura matter when cutting the bone and to prevent damaging the brain during dissection.

Key Words: Armored brain; Calcification; Chronic subdural hematoma

\section{INTRODUCTION}

Chronic subdural hematoma (CSDH) is an encapsulated collection of old blood, which is mostly or completely in the liquid state, and is located between the dura mater and arachnoid $^{1,7)}$.

The prognosis of CSDH varies widely in the literature. Most patients require surgery; in cases not necessitating urgent management, the progressive increase in volumes mandates surgical treatment. In 5.3\% of cases, the hematoma resolves spontaneously and is rarely calcified ${ }^{8)}$. Cases of encasement of the brain by massive calcified CSDH are known as "armored brain"10) These cases are rare and account for $0.3 \%$ to $2.7 \%$ of cases ${ }^{11,14)}$. In this study, we describe a case with calcification of a 38-yearold CSDH, with a ventriculoperitoneal (VP) shunt, and which resembled the skull.

\section{CASE REPORT}

\section{Patient Information and History}

The patient was a 38-year-old woman with a chief complaint of right-sided visual disturbance since the day before hospitalization. Her mental status was alert and the motor power in both extremities was normal. Since her VP shunt was placed in 1982 at the age of one year, she underwent VP shunt catheter placements on 3 occasions in 1984 (3 years of age), 1991 (10 years of age), and 2013 (32 years of age), and finally, had 1 shunt catheter on each side. She had no history other than that of brain surgery and was not taking any medications. Prior to the surgery, the blood levels of potassium and calcium were within the normal range, at $3.5 \mathrm{mEq} / \mathrm{L}$ and $9.1 \mathrm{mg} / \mathrm{dL}$, respectively.

On computed tomography (CT) and magnetic resonance imaging (MRI), a planum sphenoidale meningioma measuring app roximately $2 \mathrm{~cm}$ and compressing the optic chiasm was observed. Diffuse irregular thickened calcification with thickened reactive pachymeningeal enhancement was observed bilaterally along the cerebral convexity (Fig. 1).

Craniotomy with the removal of the brain mass was performed on hospital day 12, as no improvement was observed.
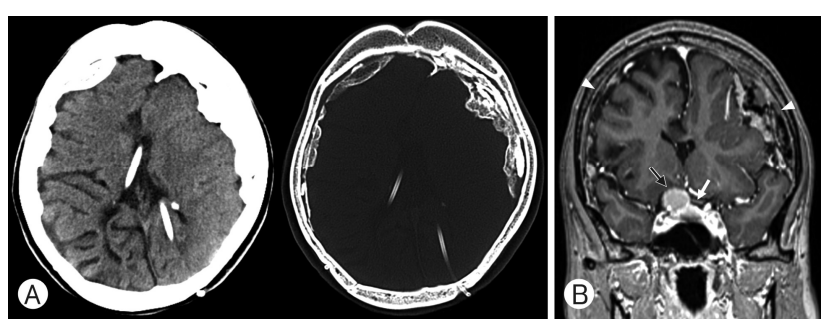

Fig. 1. (A) Computed tomography showing bilateral calcififed chronic subdural hematoma with diffuse brain atrophy. (B) Magnetic resonance imaging showing the strongly enhanced homogeneous sphenoid planum mass measuring $1.8 \times 2.0 \times 1.8 \mathrm{~cm}$ (white arrow: optic chiasm, black arrow: meningioma compressing the right optic chiasm). 


\section{Operation}

A right pterional approach was used to remove the meningioma. After piercing the zygomatic process of the frontal bone using a key burr-hole, 2 additional burr-holes were drilled into the frontal and temporal area, and craniotomy was performed using Midas $^{\circledR}$ (Medtronic, MN, USA). The dura was tightly compressed between the skull and calcific mass; the composite skull-dura calcific mass therefore had to be drilled simultaneously during craniotomy (Fig. 2).

The meningioma was compressing the second cranial nerve and optic chiasm; the operation was completed after duroplasty using a Neuro-Patch ${ }^{\circledR}$ (B. Braun Melsungen AG, Melsungen, Germany).

\section{Postoperative Course}

Postoperative hemorrhage was observed with CT in the surgical bed in the right frontal lobe. On the third day after surgery, the hemorrhage was absorbed and the brain showed some expansion. On day 14 after surgery, the density of the postoperative hemorrhage decreased significantly, and the
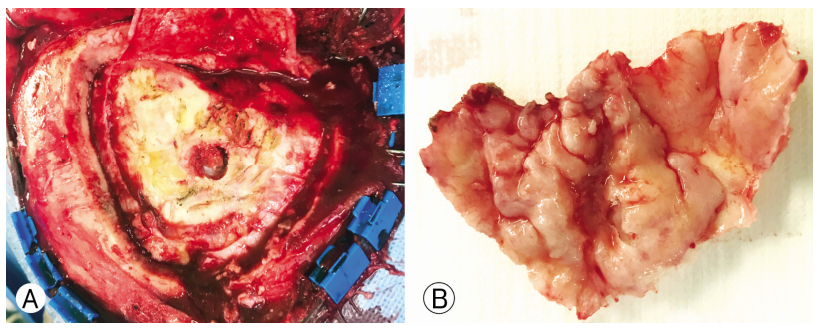

Fig. 2. (A) A calcified lesion similar to the skull is seen in the inferior aspect of the dura; a burr-hole was drilled using a neuroblade for removing the meningioma. (B) The large calcified chronic subdural hematoma known as "armored brain" demonstrating the shapes of the gyri and sulci inside the calcific mass.
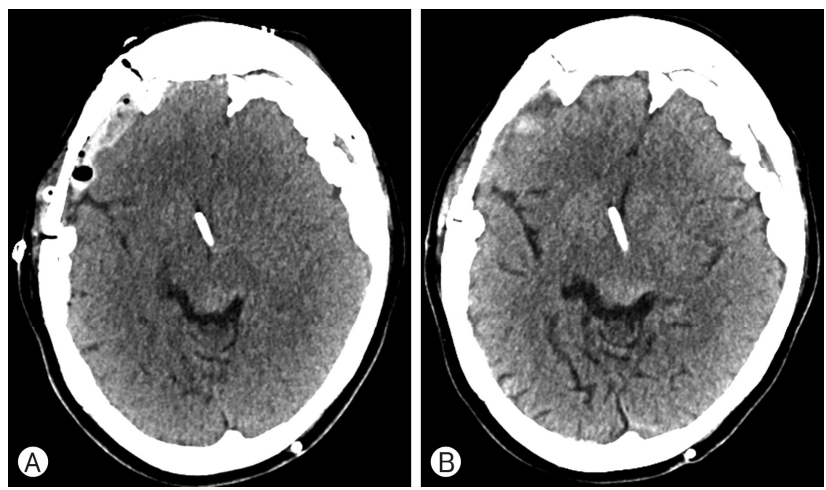

Fig. 3. (A) Right after surgery: postoperative hemorrhage in the right frontal lobe. (B) Day 14 after surgery: postoperative hemorrhage density was decreased significantly and the brain showed much expansion. patient was discharged after the evaluation improved visual disturbance (Fig. 3).

\section{DISCUSSION}

$\mathrm{CSDH}$ is a large hematoma and should be treated surgically in the presence of a mass effect; the symptoms are usually resolved on burr-hole drainage and the establishment of a closed drainage system. In $\mathrm{CSDH}$, partial craniotomy may be performed in multilobulated and multiseptate cases as well as calcified hematomas ${ }^{12)}$. Conversely, if the hematoma is small and there is no mass effect, observation during the period of resorption is appropriate.

Calcified CSDH may encase the brain hemispheres; Ludwig et al. ${ }^{10)}$ coined the term "armored brain" for this condition. While CSDH is a common disease, calcifications and ossifications are rarely observed. Calcifications and ossifications are usually observed in childhood, but have been reported in all age groups ${ }^{4)}$.

In particular, bilateral subdural calcified masses are observed after trauma and in meningitis, whereas complications of chronic shunting that result in hydrocephalus are rare ${ }^{4,6,17)}$. Other possible causes need to be investigated, and the caregiver should be aware that shunt with CSDH can cause calcification.

The underlying mechanism of calcification remains poorly understood $^{13)}$, and certain metabolic and vascular factors have been implicated ${ }^{15)}$. Boyd and Merrell ${ }^{3)}$ pointed out metabolic factors, for example parathyroid disorders, as one of the causes to calcification. Afra ${ }^{2)}$ demonstrated that potential vascular factors are absorption and circulation disorders as well as thrombosis. The time it takes for simple CSDH formation to develop into calcification varies from 6 months to several years ${ }^{5)}$. Accordingly, regular examinations are required for early detection. However, in this case, the patients were not examined for a long period of time, and thus the time course of calcification is unknown.

In 2012, Taha ${ }^{16)}$ reviewed 18 cases in which CSDH calcification appeared after shunt insertion and suggested that surgical intervention should be limited to cases with progressive neurological deficits or increased intracranial pressure. Among the 18 patients, 8 underwent surgery, and exhibited improved symptoms.

The course varies between patients, and asymptomatic cases often progress. In the case of neurological symptoms or in young children, radical curative craniotomy and complete removal of the calcification are often necessary. The course of "armored brain" is often asymptomatic; however, it may progress slowly. Neurologic symptoms such as gait disturbance, chronic headache, deterioration of vision, seizures, and dysphasia may be observed ${ }^{4,13)}$. In this study, patients exhibited no symptoms of $\mathrm{CSDH}$, but the calcified $\mathrm{CSDH}$ had to be removed due to surgical tumor resections.

It is important to remove the inner membrane of the hema- 
toma while operating on massive calcified CSDHs. Owing to severe adhesions with the pia mater, the dissection must be performed under direct vision. The brain may be more easily injured by heat than by steel burr-holes, and dural stripping using a diamond burr-hole is known to be useful ${ }^{9)}$.

Cumulative data from similar cases are needed to improve the understanding on this condition.

\section{CONCLUSION}

We have reported a rare case of calcification in a $\mathrm{CSDH}$ resulting from shunt insertion. Despite the absence of neurological symptoms, this patient required open craniotomy to remove the calcification due to brain tumor. In patients with shunts, $\mathrm{CSDH}$ persists after the previous shunt surgery and is believed to lead to calcification. During the operation, the mass appeared as hard as bone, and care must be taken not to cut the dura with instruments such as NeuroBlade.

\section{CONFLICTS OF INTEREST}

No potential conflict of interest relevant to this article was reported.

\section{REFERENCES}

1. Adhiyaman V, Asghar M, Ganeshram KN, Bhowmick BK: Chronic subdural haematoma in the elderly. Postgrad Med J 78: 71-75, 2002

2. Afra D: Ossification of subdural hematoma. Report of two cases. J Neurosurg 18:393-397, 1961

3. Boyd DA, Merrell JP: Calcified subdural hematoma. J Nerv Ment Dis 98:609-617, 1943

4. Dammers R, ter Laak-Poort MP, Maas AI: Neurological picture. Armoured brain: Case report of a symptomatic calcified chronic subdural haematoma. J Neurol Neurosurg Psychiatry 78:542543, 2007
5. Debois V, Lombaert A: Calcified chronic subdural hematoma. Surg Neurol 14:455-458, 1980

6. Garg K, Singh PK, Singla R, Chandra PS, Singh M, Satyarthhe GD, et al.: Armored brain-massive bilateral calcified chronic subdural hematoma in a patient with ventriculoperotoneal shunt. Neurol India 61:548-550, 2013

7. Horikoshi T, Naganuma H, Fukasawa I, Uchida M, Nukui H: Computed tomography characteristics suggestive of spontaneous resolution of chronic subdural hematoma. Neurol Med Chir (Tokyo) 38:527-532; discussion 532-523, 1998

8. Kim HC, Ko JH, Yoo DS, Lee SK: Spontaneous resolution of chronic subdural hematoma: Close observation as a treatment strategy. J Korean Neurosurg Soc 59:628-636, 2016

9. Kondo S, Okada Y, Iseki H, Hori T, Takakura K, Kobayashi $\mathrm{A}$, et al.: Thermological study of drilling bone tissue with a highspeed drill. Neurosurgery 46:1162-1168, 2000

10. Ludwig B, Nix W, Lanksch W: Computed tomography of the "armored brain". Neuroradiology 25:39-43, 1983

11. Moon KS, Lee JK, Kim TS, Jung S, Kim JH, Kim SH, et al.: Contralateral acute subdural hematoma occurring after removal of calcified chronic subdural hematoma. J Clin Neurosci 14:283286, 2007

12. Oda S, Shimoda M, Hoshikawa K, Shiramizu H, Matsumae M: Organized chronic subdural haematoma with a thick calcified inner membrane successfully treated by surgery: a case report. Tokai J Exp Clin Med 35:85-88, 2010

13. Petraglia AL, Moravan MJ, Jahromi BS: Armored brain: A case report and review of the literature. Surg Neurol Int 2:120, 2011

14. Sharma RR, Mahapatra A, Pawar SJ, Sousa J, Athale SD: Symptomatic calcified subdural hematomas. Pediatr Neurosurg 31: 150-154, 1999

15. Spadaro A, Rotondo M, Di Celmo D, Simpatico S, Parlato C, Zotta DC, et al.: Bilateral calcified chronic subdural hematoma. Further pathogenetic and clinical consideration on the so-called "armored brain". J Neurosurg Sci 31:49-52, 1987

16. Taha MM: Armored brain in patients with hydrocephalus after shunt surgery: Review of the literatures. Turk Neurosurg 22: 407-410, 2012

17. Viozzi I, van Baarsen K, Grotenhuis A: Armored brain in a young girl with a syndromal hydrocephalus. Acta Neurochir (Wien) 159:81-83, 2017 